\title{
Introducción \\ Cuenca y las redes sociales
}

Ángel Torres-Toukoumidis

Andrea De Santis

\section{SciELO Books / SciELO Livros / SciELO Libros}

TORRES-TOUKOUMIDIS, Á., and DE SANTIS-PIRAS, A. Introducción:

Cuenca y las redes sociales. In: TORRES-TOUKOUMIDIS, Á., and DE

SANTIS-PIRAS, A., coord. Los morlacos y las redes sociales [online].

Quito: Editorial Abya-Yala, 2020, pp. 7-12. ISBN: 978-9978-10-534-4. http://doi.org/10.7476/9789978105771.0001.

\section{(c) $\underset{\mathrm{EY}}{\mathrm{B}}$}

All the contents of this work, except where otherwise noted, is licensed under a Creative Commons Attribution 4.0 International license. Todo o conteúdo deste trabalho, exceto quando houver ressalva, é publicado sob a licença Creative Commons Atribição 4.0. Todo el contenido de esta obra, excepto donde se indique lo contrario, está bajo licencia de la licencia Creative Commons Reconocimento 4.0. 


\title{
INTRODUCCIÓN Cuenca y las redes sociales
}

\author{
Ángel Torres-Toukoumidis \\ Universidad Politécnica Salesiana, Ecuador \\ https://orcid.org/0000-0002-7727-3985 \\ Andrea De Santis \\ Universidad Politécnica Salesiana, Ecuador \\ https://orcid.org/0000-0002-7441-3794
}

En tiempos de pandemia, las redes sociales se han posicionado como las principales plataformas de entretenimiento, acrecentando de igual modo su uso en otros propósitos como es el caso del educativo (Torres-Toukoumidis \& De Santis, 2020a). En tanto, reconociendo la importancia de estos espacios de interacción, los estudiantes de Comunicación, en la asignatura Investigación de la Comunicación impartida en segundo ciclo por la Universidad Politécnica Salesiana ha compendiado un análisis sobre el uso de las redes sociales en diferentes contextos, generando una visión holística sobre su implicación en la ciudad de Cuenca.

Si bien la Universidad Politécnica Salesiana tiene sedes en Quito, Guayaquil y Cuenca, el estudio se concentró 
en Cuenca por tres razones, la primera claramente porque la asignatura era impartida en dicha sede, la segunda, porque las limitaciones de desplazamiento generadas por la Covid-19 dificultaban la accesibilidad directa a la información de las otras dos sedes y en tercera instancia, la Alcaldía de Cuenca, a través de Camila Corral, Coordinadora del Proyecto Editorial y de Fomento a la Lectura de la Dirección Municipal de Cultura, Recreación y Conocimiento apoyó el accionar y desarrollo de esta iniciativa.

La obra combina diferentes enfoques cualitativos y cuantitativos permitiendo así abordar con múltiples diseños y herramientas metodológicas destacando ciberetnografías, investigación-acción, estudios descriptivos y narrativos que utilizaron entrevistas en profundidad, documentación, encuestas entre otras herramientas de recopilación de datos. Específicamente, el libro se organiza en 11 capítulos estructurados de la siguiente manera:

El primer capítulo, "El antes y después de las redes sociales en Cuenca" realizado por Adriel Shicay-Quezada y Camila Villacís-Mendoza, aplican un diseño narrativo para determinar la incidencia secuencial de Facebook en la comunicación interpersonal, demostrando la aceptación de los cuencanos hacia esta plataforma como herramienta de uso diario, debido a sus facilidades comunicativas, su simplicidad de manejo y su versatilidad superando con creces a los canales tradicionales de comunicación.

Verónica Sigcha-Pacheco, Diego E. Vintimilla-León y Jonnathan Morocho-García elaboraron el capítulo de- 
nominado "Dicotomía rural-urbana sobre el uso de las redes sociales en Cuenca-Ecuador", en el que adaptaron un estudio cuantitativo descriptivo correlacional sobre las redes sociales en la dicotomía rural-urbana asentando que WhatsApp se utiliza más que Facebook.

En complemento, el tercer capítulo, "Lo rural y urbano: influencia de las redes sociales" realizado por Jasson Marca-Quituizaca, Alex Mejía-Loza y Christian Picón- Cedillo profundizan en este contexto desde el punto de vista más general, argumentando el despunte de TikTok en el contexto urbano.

Seguidamente, Amanda Guzmán-Heras y Tábata Figueroa-Sacoto publican "Redes sociales en jóvenes y adultos mayores cuencanos" en el que llevaron a cabo 50 entrevistas en profundidad haciendo eco la desigualdad evidenciada por la comparativa entre las personas mayores y jóvenes cuencanos en cuanto al conocimiento y uso de las redes sociales.

El próximo capítulo, "Lenguaje cuencano en las redes sociales" elaborado por Evan Gabriel Arias-Sánchez, se enfoca en el empleo de coloquialismos y spanglish por usuarios en cuentas de Facebook propiamente cuencanas, demostrando que pese a la masiva migración digital potencial aculturación, el lenguaje aun no se encuentra amenazado por cambios drásticos producidos por el influjo de las nuevas generaciones.

En el sexto capítulo, Viviana Calle-Salinas y Ximena Méndez-Calle han redactado "Influencers en la ciudad 
10

de Cuenca”, en el cual han analizado perfiles mediáticos observando las estrategias de comunicación en Instagram demostrando su importancia para las marcas en el entorno publicitario.

Continuando con los capítulos, se encuentra "Cultura cuencana y redes sociales" en el que Freddy Parra-Sigüencia, Johny Cabrera-Ruiz y Paolo Romero-Arévalo demuestran el uso de las redes sociales para enaltecer la difusión de la cultura de Cuenca mediante la incursión de herramientas audiovisuales.

En el octavo capítulo, Jhonathan Cuesta-Cuesta y Esteban Calderón-Méndez se dedicaron a entretejer la situación del comercio cuencano y las redes sociales, exhibiendo la relevancia del comercio electrónico generada por las fans page de Facebook, en el que se denota una flexibilización de los procesos de compraventa y aumento de la promoción de su oferta de productos y servicios.

Wendy Pugo-Arévalo y su compañera, Melissa Mora-Gordillo ordenan de manera coherente un análisis exploratorio sobre la práctica comunicativa de los cuencanos en el exterior, recalcando el uso de las redes sociales para satisfacer la necesidad de interacción y contacto virtual con sus familiares, además de escuchar noticias sobre su ciudad de nacimiento.

En su contraparte, la otra cara de la moneda fue otorgada por Mishell Jiménez- Pineda y Cristopher Pesantez-Maxi, quienes se orientaron a conocer la posición de los extranjeros en Cuenca respecto a las redes sociales, evi- 
denciando patrones similares al capítulo anterior, donde la mayoría se conecta para ver noticias, trabajar y contactar a sus conocidos y parientes.

Para culminar, un capítulo simbólico es "El futuro de las redes sociales en Cuenca" realizado por Fabrizzio Andree Sánchez-García y Nicolás Moscoso-Loaiza, quienes consultaron a 15 expertos de las carreras de Comunicación e Informática manifestando en general, la prospectiva de optimización de las redes sociales actuales, el cual será aprovechado por la publicidad para maximizar el desarrollo económico de la ciudad.

Estos 11 capítulos buscan complementar una colección que inició al principio del 2020 con el libro titulado: "Cuando los instagrammers son los adultos" (Torres-Toukoumidis \& De Santis, 2020b) en el que participaron estudiantes de carrera de Comunicación en la asignatura "Investigación de la Comunicación" de la Universidad Politécnica Salesiana, sede Cuenca.

Admitimos que lo vivido en el ciclo anterior con esa obra ha sido sumamente fructífera para los estudiantes, de allí que esta vez, se extrapole el proceso hacia otra investigación manteniendo los mismos propósitos: proveer una experiencia simulada del modus vivendi académico y adentrarse en la aprehensión de conocimientos relacionados con la investigación, conociendo así las formas de sistematizar la realidad y generar resultados a partir de los mismos. En definitiva, en nombre de todo el equipo, esta obra es un regalo que le hacemos a Cuenca y a todos sus ciudadanos por la conmemoración de su Bicentenario de Independencia. 
Ángel Torres-Toukoumidis, Andrea De Santis-Piras (Coordinadores)

12

\section{Bibliografía}

Torres-Toukoumidis, A., \& De Santis, A. (2020a). YouTube y la comunicación del siglo XXI. Ciespal.

(2020b). Cuando los instagrammers son los adultos. Abya-Yala. 